This Section of Epidemiology and Psychiatric Sciences appears in each issue of the Journal to stress the role of the epidemiological approach to promote advances in the field of clinical psychopharmacology, with a particular attention to controversial findings. The ultimate aims are to help develop a more critical attitude towards the results of research studies published in the international literature, to promote original research projects with higher methodological standards, and to implement the most relevant results of research in every-day clinical practice. These contributions are written in house by the journal's editorial team or commissioned by the Section Editor (no more than 1000 words, short unstructured abstract, 4 key-words, one Table or Figure and up to ten references).

\title{
Comparative effectiveness of long-acting antipsychotics: issues and challenges from a pragmatic randomised study
}

\author{
G. Ostuzzi* and C. Barbui
}

Department of Public Health and Community Medicine, Section of Psychiatry, University of Verona, Verona, Italy

\begin{abstract}
Although long-acting antipsychotics are widely used in individuals with psychotic disorders, it is unclear which long-acting preparation should be considered as first-line treatment in clinical practice. In this commentary, the main strengths and weaknesses of a recently published pragmatic randomised study comparing long-acting paliperidone palmitate $v$. long-acting haloperidol decanoate are briefly analysed.
\end{abstract}

Received 14 August 2015; Accepted 6 October 2015; First published online 30 October 2015

Key words: Effectiveness, long-acting antipsychotics, pragmatic trials, psychosis.

As the problem of both hidden and overt nonadherence to medications is a major concern in mental health, particularly in patients with psychotic disorders (Nosé et al. 2003), intramuscular long-acting formulations of antipsychotics have been developed and are currently widely used in clinical practice (Haddad et al. 2014). Despite some disadvantages, including pain on the injection site, lack of flexibility in dose adjustments and the perception of stigma and coercion (Brissos et al. 2014), these formulations allow a complete tracking of drug assumption and significantly lower the risk of self-medication and harmful drug use (Narasimhan et al. 2007; Brissos et al. 2014). Long-acting formulations may also prevent adverse events and relapse due to sudden drug interruptions (Moncrieff, 2006).

\footnotetext{
* Address for correspondence: Dr G. Ostuzzi, Department of Public Health and Community Medicine, Section of Psychiatry, University of Verona, Piazzale L.A. Scuro, 10 - 37134 Verona, Italy.

(Email: giovanni.ostuzzi@gmail.com)
}

Clinically, the choice of which long-acting formulation should be considered as first-line intramuscular treatment is very challenging. It might be assumed that current evidence on the comparative efficacy of oral antipsychotics can be directly applied to the corresponding long-acting drugs, as the active ingredients are the same. However, this assumption may be rather approximate, as several pharmacokinetic differences (absorption, distribution, half-life and percentage of receptor occupancy over time) may be responsible for substantial differences in terms of efficacy and acceptability between oral and long-acting formulations of the same molecule (Moncrieff, 2006; Meyer, 2013). Ideally, therefore, a less approximate hierarchy of efficacy and acceptability might be based on direct headto-head comparisons between different long-acting formulations. These studies, however, are virtually absent, as drug companies have no interest in putting long-acting formulations in direct competition.

However, with the aim of assessing the usefulness of long-acting formulations in the real-world of 
psychiatric practice, a recent randomised trial compared long-acting paliperidone palmitate with longacting haloperidol decanoate (McEvoy et al. 2014). About 300 individuals with schizophrenia or schizoaffective disorder were recruited from 22 clinical sites in the USA. Patients were enrolled if they were at risk of relapse on the basis of a clinical history of noncompliance and/or drug abuse. Interestingly, this judgment was based on implicit clinical expertise and not on explicit operational criteria. Patients were randomly allocated to intramuscular injections of haloperidol decanoate 25 to $200 \mathrm{mg}$ or paliperidone palmitate 39-234 mg every month for as long as 24 months of double-blind treatment. The primary outcome was efficacy failure at 24 months of follow-up, assessed as a composite measure that included psychiatric hospitalisation, a need for crisis stabilisation, a substantial increase in frequency of outpatient visits, a clinician's decision that oral antipsychotic could not be discontinued within 8 weeks after starting the longacting injectable antipsychotics, or a clinician's decision to discontinue the assigned long-acting injectable due to inadequate therapeutic benefit. A committee of three psychiatrists, blinded to the intervention allocation, appraised and discussed these clinical elements in order to produce a dichotomous judgment, namely the presence or absence of efficacy failure.

The study did not find any statistically significant difference between the two groups in terms of efficacy failure rates. The comparison of rating scale scores and sensitivity analyses confirmed this finding. With regards to tolerability outcomes, only some of the expected differences between the two antipsychotics were highlighted. Paliperidone induced more sialorrhea and weight gain, even though other metabolic indices were not different between the two groups. No differences in terms of extrapyramidal symptoms were found between the two groups, with the only exception of akathisia, which was significantly more common in the haloperidol arm. Patients taking haloperidol showed a greater need for anti-parkinsonism medications, which suggests that extrapyramidal symptoms occurred more often in this treatment group. The incidence of tardive dyskinesia was also higher in the haloperidol arm (15.4 v. 10.6\%), but the difference was not statistically significant. Paliperidone was more likely to increase blood prolactin levels, but significant differences in terms of sexual dysfunction, gynecomastia or galactorrhea were not found.

The design of this study is an original attempt to overcome the main limitations of standard explanatory randomised studies. These studies typically (a) apply strict inclusion criteria, selecting sub-populations of patients that are rare in routine care; (b) administer treatments so rigidly as to further remove the practice from reality; and (c) measure outcomes that are of questionable importance (Purgato et al. 2015). By contrast, in the present study, simple and pragmatic inclusion criteria, and flexible dose regimens, were employed. Furthermore, the main outcome measure was based on some pre-defined clinical aspects rather than on rating scales that are seldom used in day-today practice. This particular choice was thought to better resemble ordinary clinical reasoning and judgment. As a consequence of these broad inclusion criteria and methodological features, a considerable number of patients from community clinical settings were enrolled, and a very long follow-up period was planned. As the aim was not to get a new medicine into the market, but to answer a clinically relevant question, this trial received funding from a public entity (the US National Institute of Mental Health).

However, the pragmatic design of this study has also disadvantages, as pragmatism may decrease internal validity (Purgato et al. 2015). Selecting patients on the basis of an implicit judgment of being at risk of relapse and likely to benefit from a long-acting injectable antipsychotic inevitably introduces some degree of subjectivity into the recruitment process, as different doctors may have different attitudes and views on who may benefit from long-acting antipsychotics. Therefore, the sample of included patients may ultimately be rather heterogeneous in terms of several characteristics including poor adherence, which is the main reason for prescribing these formulations. Similarly, the measurement of the primary outcome, which is strongly focused on clinicians' expertise and based on different measures, raises reproducibility and reliability issues. It would therefore be of interest to ascertain whether more strict inclusion criteria and less pragmatic efficacy measure would have produced a more accurate treatment estimate. This would have made the interpretation of this negative trial easier: as the authors noted, the confidence interval around the estimate does not rule out the possibility of a clinically meaningful advantage with paliperidone palmitate, which means this study failed to demonstrate the two treatments are clinically similar.

Another consideration is that pragmatism cannot be applied to each single aspect of the study. Doubleblindness, for example, is clearly in contrast with a real-world attitude, which would imply knowledge of which treatment is given and the possibility of adjusting the dose according to clinical needs. By contrast, as this study employed a double-blind approach, clinicians received instructions on how to manage blind treatments in terms of recommended therapeutic dose, titration schedule and use of supplemental oral antipsychotics and anti-parkinsonism drugs. Clearly, some of these clinical procedures may be considered rather artificial and far from everyday practice. 
This trial, in line with previous findings (Lieberman et al. 2005; Leucht et al. 2013), contributes to reconsider the previous assumption of a substantial difference between newer and older antipsychotic drugs, and expands this appraisal to long-acting formulations. It has the valuable merit of comparing two commonly used long-acting drugs, aiming at better informing psychiatric practice. As discussed, the main weaknesses of these studies are the low accuracy and reliability of the procedures for selecting patients and assessing outcomes, which may have lowered the internal validity of the study. However, it should be recognised that there is an intrinsic complexity in planning a study that simultaneously is expected to: (a) include a truly representative population, (b) frame clinically relevant outcomes, (c) minimally interfere with ordinary practice and (d) keep a high standard in terms of internal validity. Pragmatism is a relative concept and even pragmatic trials are not immune to selection bias, as they naturally tend to select patients who are at least partially adherent to clinician's indications (Brissos et al. 2014), a real paradox in this case as individuals at risk of non-adherence were the main target population.

\section{Financial Support}

No financial support was received for this paper from any funding agency, commercial or not-for-profit sectors.

\section{Conflict of Interest}

None.

\section{References}

Brissos S, Veguilla MR, Taylor D, Balanzá-Martinez V (2014). The role of long-acting injectable antipsychotics in schizophrenia: a critical appraisal. Therapeutic Advances in Psychopharmacology 4, 198-219.

Haddad PM, Brain C, Scott J (2014). Nonadherence with antipsychotic medication in schizophrenia: challenges and management strategies. Patient Related Outcome Measures 5, 43-62.

Leucht S, Cipriani A, Spineli L, Mavridis D, Örey D, Richter F, Samara M, Barbui C, Engel RR, Geddes JR, Kissling W, Stapf MP, Lässig B, Salanti G, Davis JM (2013). Comparative efficacy and tolerability of 15 antipsychotic drugs in schizophrenia. A multiple-treatments metaanalysis. Lancet 382, 951-962.

Lieberman JA, Stroup TS, McEvoy JP, Swartz MS, Rosenheck RA, Perkins DO, Keefe RSE, Davis SM, Davis CE, Lebowitz BD, Severe J, Hsiao JK (2005). Effectiveness of antipsychotic drugs in patients with chronic schizophrenia. New England Journal of Medicine 353, 1209-1223.

McEvoy JP, Byerly M, Hamer RM, Dominik R, Swartz MS, Rosenheck RA, Ray N, Lamberti JS, Buckley PF, Wilkins TM, Stroup TS (2014). Effectiveness of paliperidone palmitate vs haloperidol decanoate for maintenance treatment of schizophrenia: a randomized clinical trial. JAMA 311, 1978-1987.

Meyer JM (2013). Understanding depot antipsychotics: an illustrated guide to kinetics. CNS Spectrums 18 (Suppl. 1), 58-67, quiz 68.

Moncrieff J (2006). Does antipsychotic withdrawal provoke psychosis? Review of the literature on rapid onset psychosis (supersensitivity psychosis) and withdrawal-related relapse. Acta Psychiatrica Scandinavica 114, 3-13.

Narasimhan M, Un Pae C, Masand N, Masand P (2007). Partial compliance with antipsychotics and its impact on patient outcomes. International Journal of Psychiatry in Clinical Practice 11, 102-111.

Nosé M, Barbui C, Tansella M (2003). How often do patients with psychosis fail to adhere to treatment programmes? A systematic review. Psychological Medicine 33, 1149-1160.

Purgato M, Barbui C, Stroup S, Adams C (2015). Pragmatic design in randomized controlled trials. Psychological Medicine 45, 225-230. 